\title{
Commentary on the Continued Investigational Status of DBS for Psychiatric Indications
}

\author{
Robert J. Coffey ${ }^{a}$ Stanley N. Caroff ${ }^{b}$

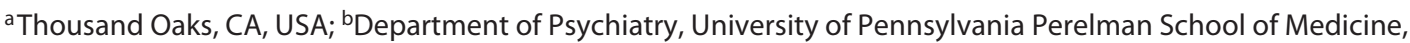 \\ Philadelphia, PA, USA
}

\section{Keywords}

Clinical trial · Deep brain stimulation · Depression · Ethics ·

Obsessive-compulsive disorder · Psychosurgery

\begin{abstract}
Behavioral disorders exact a tragic toll on patients, families, and society. Consequently, the search for better treatments is a public health priority. Recent research promises to lead to advances in psychiatric treatment that may include implantation of deep brain stimulation (DBS) devices. In this commentary, the authors discuss how promising results from initial pilot studies of DBS in treatment-resistant depression (TRD) were not validated in 2 randomized, controlled, multicenter trials. Reliance on pilot data may have contributed to the selection of primary efficacy endpoints that were not achieved, and to the underestimation of adverse events and device-related complications. Published data on the population prevalence of affective disorders also may have led sponsors to overestimate the number of patients with TRD who were candidates for DBS therapy. Consequently, a more complete discussion of certain aspects of the depression trials may allow a realistic appraisal of the clinical and ethical situation of DBS therapy for TRD in a US regulatory context. A US regulatory perspective also may clarify the clinical research and reimbursement consequences of the Humanitarian Device Exemption (HDE) approval status of DBS for obsessive-compulsive
\end{abstract}

disorder (OCD). Retrospective analyses akin to failure modes and effects analysis in engineering may clarify unexpected results in the DBS depression trials. Recent research suggests that subject selection in future trials may be augmented by advanced neuroimaging methods. For the present, the noncommercial research status of DBS to treat depression and the HDE status for OCD appear likely to remain in place.

(c) 2022 S. Karger AG, Basel

\section{Introduction and Background}

"Because half-a-dozen grasshoppers under a fern make the field ring with their importunate chink, ... pray do not imagine that those who make the noise are the only inhabitants of the field; ..." Edmund Burke.

(https://www.oxfordreference.com/view/10.1093/ acref/9780191843730.001.0001/q-oro-ed5-00002268)

The search for a somatic cure of mental disorders has beguiled clinicians for millennia. Concepts of psychiatric disorders and treatments also reflect the contemporary medical and social zeitgeist [1]. For example, pre-enlightenment physicians condoned trephination to rid the afflicted person's brain of noxious vapors or the stone of madness. In the early 20th century, radical surgical extirpations were promoted as a cure for imagined causative sites of infection,
Correspondence to:

Robert J. Coffey, robert.coffey@ netzero.net 
followed by enthusiasm for prefrontal leukotomy ([2], https://www.nobelprize.org/prizes/medicine/1949/ summary/). Fortunately, such treatments were abandoned as scientific evidence supported the effectiveness of newer pharmacological agents.

More recently, deep brain stimulation(DBS) has emerged as a potential, contemporary, neuroscience-based surgical therapy for psychiatric disorders [3]. However, 2 industry sponsors closed formal programs toward FDA premarket approval (PMA) of DBS in patients with treatmentresistant depression (TRD) in 2011 and 2013, respectively (Medtronic, Inc. Reclaim trial with leads in the ventral capsule/ventral striatum [VC/VS] region, and the St. Jude Broaden trial with leads in the subcallosal cingulate [CG25] region) $[4,5]$. Both sponsors intended to enroll approximately 200 subjects in controlled, randomized, blinded trials, as required by the FDA for PMA for a new indication. Although some have referred to Reclaim and Broaden as pivotal trials, passage of specific intermediate endpoints was required in both programs before the FDA would allow 200 subjects to be enrolled [6-8]. Only then would the trials have achieved pivotal status sufficient to support a PMA application for TRD. The unexpected results of interim analyses required by the FDA (at 30 subjects for Reclaim; 90 for Broaden) led both sponsors to close their development programs.

Years after closure of the industry-sponsored DBSTRD trials, recent clinical publications and ethical recommendations stimulated this commentary $[9,10]$. Ingenious neurophysiological and imaging studies also have been conducted that bear upon the limitations inherent in responder analyses, and on the clinical, regulatory, and ethical issues that face DBS for TRD, in the USA $[9,11,12]$. A re-assessment of evidence from a US regulatory perspective, referenced by portions of section 21 of the Code of Federal Regulations, the Food Drug and Cosmetic Act, and as published by FDA officials may be worthwhile regarding DBS for TRD, and secondarily, regarding the Humanitarian Device Exemption (HDE) for obsessivecompulsive disorder (OCD) (Table 1) [8].

\section{Implications of the Pilot Studies for Industry- Sponsored TRD Trials}

A 1999 publication by European investigators described the effects of DBS administered to the anterior limbs of the internal capsule in 4 patients with OCD. Implantable systems were provided through an industry research support program (Medtronic, Quest) [13]. Soon after, US investigators

Continued Investigational Status of DBS

for Psychiatric Indications received FDA approval for physician-sponsored IDEs to study DBS in pilot studies of patients with psychiatric disorders - implanting approximately 6 or fewer subjects with OCD (and later, depression) per center. With respect to depression, investigators at 3 US centers reported pooled results of VC/VS DBS in 15 subjects treated under openlabel single-institution protocols with different selection criteria and DBS targets that varied over time - limitations that were disclosed in the publication [14]. Industry support included research-use-only device systems, postoperative target mapping, and clinical outcome analyses - anticipating that promising peer-reviewed pilot data would increase support for testing the experimental hypothesis, and assist in the recruitment of later phase depression trial centers and subjects $[14,15]$. Other centers in North America and Europe implanted depressed subjects with the same devices outside of the pooled analysis [16-20]. The 3 participating US centers reported $>40 \%$ improvement in depressive symptoms at 1 year and 46-56\% improvement over 6 months in DBS responders - depending on the assessment interval and outcome scale (Montgomery-Åsberg Depression Rating Scale [MADRS]; Hamilton Depression Rating Scale [HAM-D]) [21, 22]. However, during protocol development for later phase VC/VS TRD trials (feasibility and pivotal), the development team appears in retrospect to have underestimated how pilot study subject selection, absence of randomization, and unblinded assessment may have exaggerated apparent efficacy. In terms of subject selection, primary diagnoses included major depressive disorder in 14 subjects and bipolar 1 disorder in one. However, multiple comorbidities characterized pilot trial subjects including melancholic versus psychotic features, borderline and mixed personality disorders, dysthymia, panic disorder, agoraphobia, social phobia, alcohol or polysubstance dependence, eating disorders, and post-traumatic stress disorder [14]. Although some of those comorbidities became exclusion criteria in Reclaim, secondary diagnoses in that 30-subject cohort also may have confounded efficacy analyses. The overall effect was that planners appear to have applied an insufficient discount for comorbidities when they used single-center results to calculate projected, future efficacy in the more rigorously controlled multicenter trial.

Pilot study results also may have affected planning for the CG25 multicenter Broaden trial. According to the authors, "On the basis of preliminary data [23], response in $40 \%$ or greater of patients was anticipated for the group assigned to active subcallosal cingulate DBS, with response in $18.5 \%$ or fewer of patients in the control group expected [24]." Other data suggested the response rate may have 


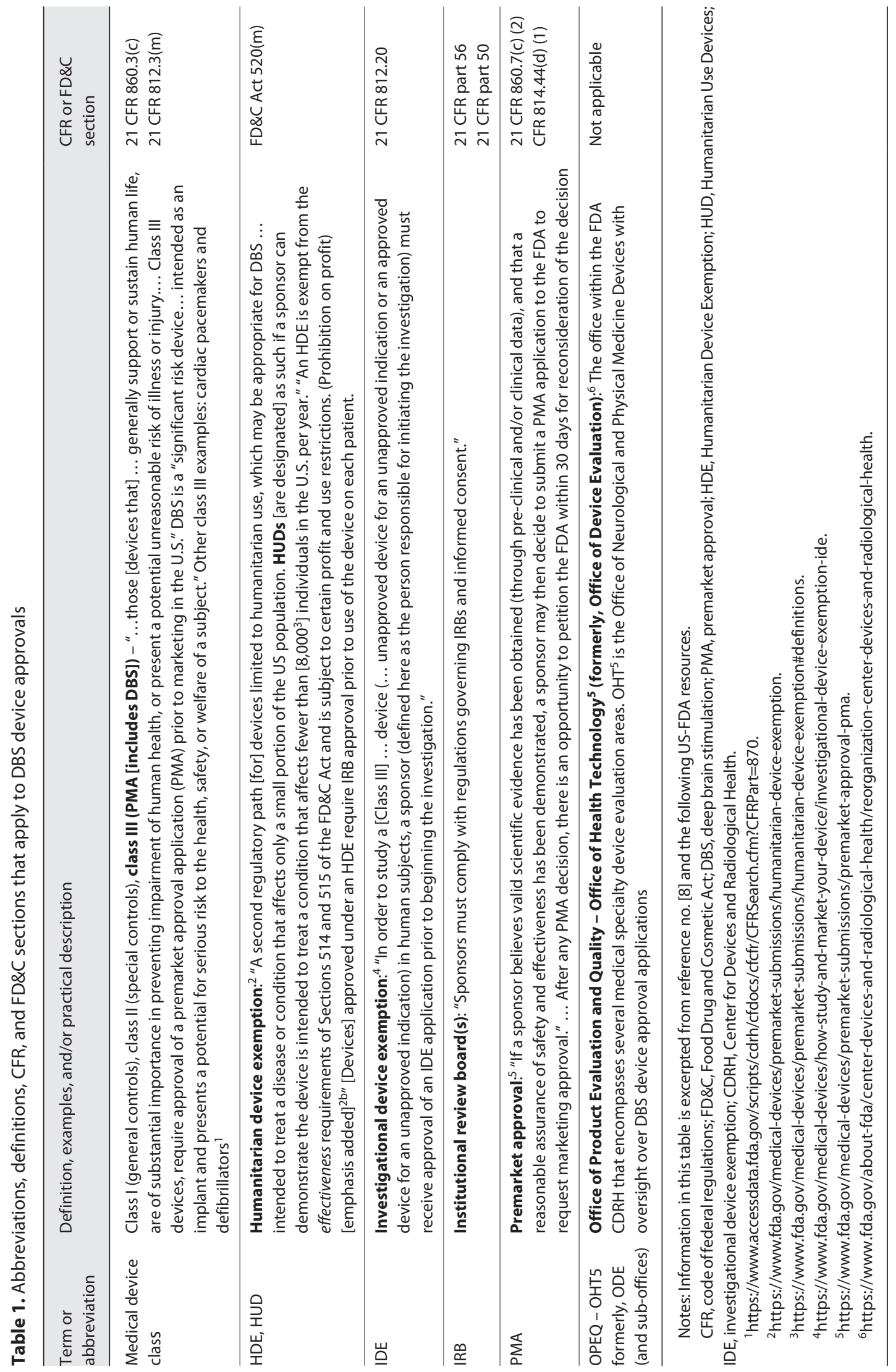


diminished as more subjects accrued: from $66.6 \%$ responders (4 of 6 ) in the first report to $55 \%$ ( 11 of 20 ) in a follow-on publication $[25,26]$. The separately reported multicenter pilot study revealed that the proportion of 21 subjects whose HAM-D scores improved by $\geq 50 \%$ had decreased from $57 \%$ at 1 month to $48 \%$ at 6 months, and to $29 \%$ at 12 months [23]. By redefining response as a $40 \%$ decrement in the HAM-D score at 12 months, the response rate more than doubled to $62 \%$. Thus, some subjects experienced fluctuating efficacy that, by some analyses, diminished over time. The effect size also varied between individuals, with standard deviations amounting to $1 / 2-3 / 4$ of the response rates $( \pm 29.8 \%$ at 2 months; $\pm 31.3 \%$ at 6 months; and $\pm 23.0 \%$ at 12 months). The rigorously controlled and blinded Broaden CG25 investigational plan nevertheless projected that more than $40 \%$ of actively treated subjects (DBS “on") would experience improvement in their MADRS score by $\geq 40 \%$ during months 4-6 of the blinded study period.

Efficacy in both sponsored trials (albeit at different targets and in different numbers of subjects) amounted to approximately half of what had been anticipated. The primary outcome measure in the VC/VS Reclaim study - defined as $\geq 50 \%$ improvement on the MADRS from baseline - revealed no difference in depressive symptoms. Less than $20 \%$ improvement was observed after 16 weeks of active or sham stimulation among the 30 subjects [4]. The Broaden trial of CG25 DBS studied 90 implanted subjects who were randomized 2:1 for 6 months of active or sham stimulation ( $n=60$ and 30 , respectively). With a $\geq 40 \%$ improvement in MADRS to qualify as a response in the CG25 trial (vs. 50\% at VC/VS), the Broaden trial yielded nearly identical results as the Reclaim trial: $19.6 \%$ improvement during months $4-6$ in both the active stimulation and control groups [5].

The US regulatory regime for class III device approval leaves no straightforward and likely-to-succeed pathway open toward approval of DBS for TRD without the active participation of a commercial sponsor that already "owns" an FDA master file that includes DBS manufacturing, technology, and approvals for other indications. Medtronic and Abbott-St Jude appear to have exited or indefinitely suspended their depression PMA program. Boston Scientific (Marlborough, MA, USA), another US-based DBS manufacturer, mentions only movement disorder topics on their Investigator Sponsored Research Program web page [https://www.bostonscientific.com/en-US/investigatorsponsored-research.html]. Suspension or closure of work by a sponsor toward PMA for a new DBS indication creates a different situation than FDA denial (nonapproval) of a completed PMA application. For example, after nonapproval of a PMA submission to treat epilepsy, additional work and data analyses after timely filing of an appeal petition (Table 1) eventually led to DBS marketing approval years later [27, 28]. To our knowledge, the DBS-TRD trial sponsors elected not to continue their PMA programs. Consequently, they did not file appeal petitions, did not seek to enroll a pivotal number of subjects, and, therefore, could not submit completed PMA applications to the FDA. FDA review, denial, petitioning, and re-evaluation did not occur. This did not necessarily foreclose future industry support in the form of devices and analytical or statistical assistance for physician-sponsored IDE research. But the present situation suggests that device makers have little immediate interest in additional investments toward completion of PMA applications for TRD.

\section{The OCD HDE: Research, Regulatory, and Commercial Considerations}

DBS for one psychiatric condition, OCD, received FDA Humanitarian Use Device (HUD) designation and HDE approval in 2009 ([29], https://www.accessdata.fda. gov/scripts/cdrh/cfdocs/cfhde/hde.cfm?id=H050003) The HDE approval and HUD designation processes summarized in Table 1 include exemption from evidence-based effectiveness requirements - meaning that FDA reviewers performed no formal, critical analysis of efficacy data comparable to a PMA application. That is why DBS devices intended for implantation in US patients with OCD carry generic HDE labeling which reads: "The effectiveness of this device for this use has not been demonstrated." Other HDE- and HUD-related requirements include IRB approval prior to implantation in each patient, a limit on the number of devices allowed to be sold per year in the US to 8,000 , and a prohibition on profit for devices sold under an HDE. Because of those regulatory restrictions, a number of investigators (predominantly, but not exclusively outside the US) criticized the sponsor's OCD-HDE application, and the FDAs HUD designation and HDE approval, respectively [29]. Research-related concerns turned out to be prescient. Despite an IRB approval requirement for each individual patient, DBS implantation under the OCD HDE does not mandate the same level of patient follow-up and uniform, protocol-driven data collection as occurred, for example, in the physiciansponsored IDE studies of DBS for OCD or depression. In addition, one investigative team reported that the proportion of candidates implanted for OCD diminished 
substantially in their research program after the HDE took effect [30]. Their review implicated denial of government (Medicare, Medicaid) or commercial insurance coverage - cited explicitly in documents contained in patients' records - as a principal barrier to implantation of otherwise suitable candidates.

Technically, HDE-HUD status is a category of FDA approval, albeit one that does not carry the same evidentiary weight as PMA. However, FDA approval of devices or drugs does not necessarily translate to insurance coverage. The sponsor and implanters already had experienced the insurance-related consequences of HDE and HUD approvals for a number of years after the DBS for dystonia HDE in 2003 [https://www.accessdata.fda.gov/scripts/ cdrh/cfdocs/cfhde/hde.cfm?id=H050003, https://www. accessdata.fda.gov/scripts/cdrh/cfdocs/cfhde/hde. $\mathrm{cfm}$ ?id=H020007]. Published coverage and payment scales, including Medicare, explicitly prioritize price over FDA approval status ("Tiers"), under: [https://www.medicare. gov/drug-coverage-part-d/what-medicare-part-d-drugplans-cover, https://www.medicare.gov/medicare-and-you, https://www.medicare.gov/drug-coverage-part-d].

Patient recruitment, therapy access, and payment matters are important, but entirely separate from the clinical data requirements for a PMA submission and FDA approval. The aggregate number of OCD cases encompassed in a 2020 review of approximately 20 years of published studies amounted to approximately 150 patients or subjects, excluding duplications [31-33]. The reviewers assessed levels of evidence that varied from level 1 to level 3 (aggregated or cohort studies) [31]; investigational sites primarily were outside the USA; subjects were implanted at different anatomical targets; and enrollment, selection, and follow-up took place under different investigational protocols. Similar features, from a US regulatory perspective, characterized a 2014 guideline (approximately 72 subjects) [34] and a substantially updated 2020 CNS guideline update (approximately 155 additional subjects) [https:// www.cns.org/guidelines/browse-guidelines-detail/deepbrain-stimulation-obsessive-compulsive-disord]. Recently reviewed OCD cases now amount to approximately 225 individuals in level 3 studies, or higher (as judged by the guidelines authors). In our estimation, and as discussed in more detail below, the features of predominantly nonUS centers, varied DBS targets, and varied research protocols - regardless of data quality or efficacy results - still comprise an insufficiently uniform, controlled, or monitored dataset for a PMA application [35-40]. Sound medical research methods, accurately reported results, and sober analyses of efficacy and safety may support physician, patient, and medical society judgments about the value of DBS to treat OCD [41]. However, absent a prospective, FDA-approved, industry-sponsored research study formally directed toward PMA - and consisting of a large single cohort enrolled into a professionally monitored dataset we believe that the likelihood of US-FDA approval of DBS to treat OCD beyond the current HDE (with attendant research and insurance limitations) remains vanishingly low [42].

During the psychiatric therapy program decision process, population estimates that led the sponsor to pursue a PMA route for TRD (candidate population too large for $\mathrm{HDE} /$ potentially large investment return) also led Medtronic to pursue the HDE route for OCD (candidate population within HDE range/low return on investment) [43]. Even with unlimited funds and participation of every active investigational site in the USA, the development team concluded that they could not recruit, implant, follow-up, and retain a sufficient number of OCD clinical trial subjects over an economically feasible time period to complete a PMA application. Given the time periods that elapsed during patient accrual and reporting in studies cited in consensus statements and practice guidelines, the decision to pursue divergent US regulatory pathways for DBS to treat OCD versus TRD appears to have been correct on strictly US regulatory and commercial terms. But contrary to expectations of the sponsors and investigators, the regulatory pathway toward PMA for TRD encountered unanticipated efficacy barriers which, in turn, led to sponsor decisions that created additional barriers for approval of the DBS depression indication.

\section{Adverse Event Reporting}

One compelling rationale for DBS treatment is the potential for suicide in TRD patients. However, none of the data in either sponsor's program suggests that suicidal ideation or suicide rates in the DBS trial cohorts differed from the overall population of similarly afflicted patients [44-47]. Trial participation neither increased nor diminished the incidence of suicidality or suicide relative to estimates in the population. Likely owing to small numbers, no formal analyses of suicidal ideation were reported in either trial. The combined aggregate numbers for suicidal ideation serious adverse events (SAEs) during the blinded phase of both trials were 3 versus zero subjects among active versus controls, respectively $(3 / 76 ; 4 \%$ [60 active, Broaden +16 , Reclaim] $)[4,5]$. Blinded phase active (DBS "on") patients in aggregate also experienced somewhat 
more "worsening depression" (Reclaim) or an "increase in depressive symptoms" (Broaden) versus controls (13/76; $17 \%$ vs. $4 / 449 \%$ [ 30 controls, Broaden +14 Reclaim]) $[4,5]$.

Apart from reporting accurate numbers, the language used to report suicidality and related events in DBS-TRD trial publications may be susceptible to misinterpretation $[4,5,25]$. Authors of an early CG25 pilot report wrote that "No significant adverse events were reported during follow-up, although 2 patients died by suicide during depressive relapses [25].” In the Broaden trial, 5 suicidalityrelated SAEs occurred, and "There were 2 deaths by suicide in the study; both were in the control group during the 6 months open-label phase [5]." A lay reader might infer that the passage "individuals in the control group" means they committed suicide while not receiving active stimulation. However, despite those subjects' assignment to the control group during the blinded study period, they were receiving active DBS when their suicides occurred. Suicidality SAEs also were reported with few details or context in the VC/VS Reclaim trial (including the openlabel extension phase) wherein "... there was one completed suicide which was deemed not to be related to the stimulation. The suicide occurred in a nonresponder who was taken off stimulation in preparation for explantation of the DBS system [4]." While the published accounts are accurate, FDA reviewers of SAE narratives in a PMA submission would adjudicate completed suicide while onstudy as broadly related to the subject's treatment. Other details in the latter individual's case raise questions about how eligibility criteria were applied to the subject's participation in the first place - including suicidality during previous treatment with an implanted vagus nerve stimulator, hypomanic episodes, and polysubstance abuse, which should have excluded trial participation. However, owing to closure of the programs without submission of complete PMA datasets, FDA review of SAEs did not occur.

Device-related complications in the DBS-TRD trials may also merit additional attention. Sponsors expect device- and procedure-related AEs to occur in DBS clinical trials at rates comparable to other approved indications. However, a disproportionately high number of surgical implant complications occurred in the Reclaim trial. The trial publication states that "... 3 subjects required lead revision for reasons other than infection [4] [emphasis added]." One lead was replaced as required per-protocol because it was outside the target region. Another subject's lead was replaced in a (sponsor-approved) deviation from the trial protocol. It was on target, and revision was not required, but the protocol deviation was requested

Continued Investigational Status of DBS

for Psychiatric Indications in an effort to convert the subject from nonresponder to responder status. "The third subject required lead revision due to high impedance readings of the original lead," which made stimulation impossible. A more complete tally of device implant errors (most classified by the sponsor, per-protocol as "technical observations") revealed that 10 patients from all 5 centers showed high lead impedance readings - an indication of mechanical damage. In 9 of 10 , the damage was visible on plain radiographs. All resulted from unexplained deviations from the surgical protocol or use of incompatible stereotactic accessories. The 10 damaged leads or connectors in this 30-subject, 5-center trial contrasted sharply with 7 comparable events in a contemporaneous 157 -subject/17 center trial for epilepsy that employed the same DBS system [27]. Absence of patient harm permitted such events to be classified other than AEs. But study personnel had to "program around" the nonfunctioning contacts during survey testing before randomization - a situation that may have affected overall trial efficacy. That is because the nonfunctioning contacts had to be excluded during the primary efficacy measure in the blinded phase (and from open-label stimulation in the continuation phase). It remains a matter of speculation whether the nonfunctioning contacts, distributed among almost one-third of the 30 -subject cohort, might have proven more efficacious than the remaining partial complement of contacts that remained available for DBS treatment.

Other AEs in Reclaim bear upon subject suitability, clinical decisions and equipoise, and published ethical recommendations. Examples include an elderly man with more than a dozen medical comorbidities who was diagnosed with worsening dementia after DBS implantation. Another was a young man with substance abuse disorder (which should have excluded enrollment) who stole a DBS programmer in order to self-stimulate. In another subject, one DBS lead was implanted off-target, and he never responded to VC/VS stimulation. The lead was not revised before trial programming according to the protocol - in part because the subject resided on the opposite coast from the implant center. At the time of pre-enrollment screening, he also had an investigational cortical stimulation device in place that was not effective. A pre-enrollment recommendation to exclude the subject was overruled. Eventually, the subject and investigators requested termination of his participation, after which the DBS system was removed (the cortical stimulation system had been explanted at enrollment), and a cingulotomy was performed. Three more subjects at 2 centers were classified in retrospect (after implantation) as "eligibility criteria not met" because their 
psychiatric medications were not kept constant during the 30-day run-in period. By chance, all 3 were randomized to the control group during the blinded period.

\section{Ethical Considerations, Perceptions, and Language}

A recent technology review contained ethical recommendations that appear to be based upon efficacy and safety assessments in DBS-TRD trial responders [10]. To quote: "Brain implant trials generally do not have provisions to ensure that patients/subjects who gain clinical benefit from the use of DBS will have access to maintenance of the device after completion of the trials.... Patients who participate in these trials have severe and treatment-resistant neuropsychiatric conditions. Axiomatically, patients with 'treatment-resistant' disorders who benefit from an experimental DBS intervention during a trial have no other effective treatment alternatives; and guaranteed provision of services and resources to assure maintenance of DBS devices upon completion of such trials is lacking. To be sure, such continued maintenance may incur significant costs." ...

The continuation of DBS in subjects from closed TRD clinical trials may be ethical under certain conditions and limitations not addressed in the quoted publication [10]. After closure of an industry-sponsored IDE trial, except under specific conditions, the sponsor's financial responsibilities end for individual subject care. However, industry sponsors - or alternatively, physicians at investigational centers - commonly open long-term continuation or follow-up (LTFU) studies that specifically limit entry to subjects from the earlier trials. LTFU studies require a separate physician- or industry-sponsored IDE application, a formal research protocol and informed consent, IRB approval and oversight, and regulatory reporting. These provide the same subject protections as the original trials and make LTFU studies distinct from so-called off-label use in ethical and regulatory terms. That still leaves open the matter of financial support. In the USA, financial support is negotiated with the industry sponsor, and may include government, private philanthropic, and/or institutional contributions. From an ethical perspective, having funding in place appears advisable before offering LTFU participation to subjects in a trial that is about to close. A potentially chaotic situation arose for some DBS research subjects (especially responders) who declined to have their systems explanted at centers that did not open LTFU studies. They, and the cohort from a center that withdrew from the Reclaim trial, had to be referred elsewhere for LTFU care [4].
The ethics-related quotation above also implies that the programs ended abruptly, and that subjects and investigators were not informed of sponsors and FDA's plans for trial closure if specified endpoints were not achieved. The Medtronic VC/VS trial investigator and informed consent agreements - and most likely, the corresponding Abbott/ St. Jude CG25 trial documents (no longer available online) [https://clinicaltrials.gov/ct2/show/NCT00617162?term= NCT00617162\&draw=2\&rank=1] - described how the clinical programs would wind down if FDA-PMA did not appear feasible [https:/clinicaltrials.gov/ct2/show/ NCT00837486? term $=$ NCT00837486\&draw $=2 \&$ rank $=1]$. Given the lack of clear benefit and the potential for devicerelated contraindications to arise during future medical care (e.g., electroconvulsive therapy, repetitive transcranial magnetic stimulation, electromagnetic interference, magnetic resonance imaging [MRI], ultrasound, diathermy, or lithotripsy) the VC/VS trial sponsor recommended that all subjects undergo complete DBS system explant at the company's expense once the trial closed. As a condition of participation, investigators and subjects signed agreements that if the program ended without DBS depression therapy moving toward FDA approval, sponsor payments would cease and insurance coverage was unlikely. Funding "services and resources to assure maintenance of DBS devices upon completion of such trials" [10] would in the absence of physician- or industry-sponsored LTFUIDE protocols described above - amount to continuation of unapproved therapy that would expose subjects to risks beyond what they had consented to originally. Without specific LTFU protocols in place, we expect the FDA would be loath to permit sponsors to continue support for implantable device therapies that did not meet endpoints specified in the PMA application. However, payment is one among several ethical issues facing future DBS depression research.

The ethical merit of any medical therapy relies upon consistency with 4 standards: beneficence, non-maleficence (closely related to beneficence), autonomy, and justice [48]. Beneficence entails "the obligation of [a] physician to act for the benefit of the patient,... [and] prevent harm" Beneficence also has "positive requirements ... not just avoiding harm, but also ... to promote their [patients] welfare [48]." The related principle of "non-maleficence ... is for the physician to weigh the benefits against burdens of all interventions and treatments, to eschew those that are inappropriately burdensome, and to choose the best course of action [48]." The beneficence standard poses ethical questions that, arguably, are intertwined with judgments about therapeutic efficacy. And under the non-maleficence 
principle, beneficial intentions alone are insufficient. In the case of DBS, responders from the Broaden or Reclaim trials who participate in LTFU studies need to invest additional time and resources, and potentially risk additional exposure to surgical and other device hazards - all without the confidence of level-1 evidence that their apparent therapy responses in open-label LTFU are due to DBS therapy. Such studies may be ethical as long as participants are well informed. Investigators, IRBs, and patients now face practical and ethical decisions that are more complex and require more information to be conveyed (e.g., trial results) than when the depression studies began.

Autonomy, the principle that "Every human being of adult years and sound mind has a right to determine what shall be done with his own body," also relies upon informed consent to disclose risks and realistic expectations [49]. There being no current PMA for depression, and uncertain benefits in light of previous trial results, suggests that respect for autonomy means US patients should be informed that study participation is largely an altruistic endeavor for research purposes.

Justice in medical ethics refers to fair and equal treatment of all persons. When expanded to society at large, "Distributive justice refers to the fair, equitable, and appropriate distribution of healthcare resources ... [that] include allotment of scarce resources ..., and allotment of time" [50]. Among those dual criteria, societal demands pose the most difficulty for DBS. The therapy is expensive, time consuming, and resource-intensive. Given the costs, personnel and resource intensity, risks, and results of the Broaden and Reclaim trials, ethical considerations pertaining to justice, especially distributive justice, also have acquired additional complexity.

Another ethical matter is how the medical community perceives the aftermath of the DBS depression trials. Some physicians may misinterpret later publications and sincerely believe that off-label use of DBS is a valid, evidence-based treatment for depression - limited only by industry, regulatory, and insurance decisions. The HDE status (and availability) of DBS for OCD may feed into such judgments by normalizing a shift away from strictly research settings in the use of DBS for psychiatric disorders. In a similar context, with respect to language, FDA required that sponsors enumerate detailed criteria to define the treatment resistant subset of the major depressive disorder population targeted for recruitment into the DBS trials. However, TRD is not defined uniformly in DSM-IV-TR, DSM-V, or among US health-care agencies ([51, 52], https://www.cms.gov/Medicare/Coverage/ DeterminationProcess/downloads/id105TA.pdf, https://www.ahrq.gov/sites/default/files/wysiwyg/research/ findings/ta/topicrefinement/trdepression-protocolamendment.pdf). Despite precise definitions within trial protocols, the notion of treatment resistance subsequently appears to have acquired a semantic life of its own that is no longer bound by protocol-specific constraints. Appearance in the peer-reviewed medical literature on DBS for depression and other psychiatric disorders of value-loaded expressions such as "cutting edge," "life-saving," "lifetransforming," and "desperate to get better" signals to readers that debate or disagreement equates with opposition to medical progress and possibly worse - insensitivity to patient needs [7, 10, 53, 54]. More important, the conflation of treatment resistance with "no other treatment alternatives," when presented to patients in those words, potentially carries the risk of exposing them to excessive persuasion to the point of undue influence, for example, if DBS were presented as a treatment (or the only treatment) of last resort [10]. That aside, at least one pharmacological treatment that did not exist when the Broaden and Reclaim trials were underway is now FDA-approved for TRD. Nasally administered esketamine in conjunction with oral antidepressant medication has shown evidence for efficacy with short onset in TRD (including with acute suicidal ideation) and lasting 4-6 months - comparable to the duration of the blinded phase DBS trial periods [55]. The approved labeling cautions that nasal esketamine "does not preclude the need for hospitalization if clinically warranted, even if patients experience improvement after an initial dose ..." [https://www.janssenlabels.com/packageinsert/product-monograph/prescribing-information/ SPRAVATO-pi.pdf].

\section{DBS Depression Research in the Future}

In the absence of industry sponsorship for US-FDA approval, what might a next generation of progress-oriented and ethically acceptable DBS depression research look like? Taking as an example the DBS-TRD trials that yielded unexpected results, we would consider starting with an "after action report" or failure modes and effects analysis that focuses on subject selection. To our knowledge, a systematic, independent audit of previous clinical trial referral records, psychiatric (and other) diagnoses, and, ultimately, trial eligibility has not yet been performed. The rationale for such a post hoc undertaking is that adverse event reviews belatedly revealed subjects who probably should not have been selected for participation. Despite limitations of hindsight bias, it could be informative for an expert panel 
blinded to individual subject efficacy results, to identify imperfectly recruited subjects. We expect that individuals identified in the adverse events section above (and possibly others) - all of whom withdrew or were nonresponders would be flagged for exclusion. Aside from answering the "who did we really treat"? question, the resulting cohorts could be matched with their efficacy outcomes. Such analyses are not permissible in a regulatory submission, but an outcome assessment by protocol-intended eligibility may identify the DBS candidates who were most likely to succeed, leading to better subject selection in the future.

Other research already underway has explored refinements in targeting based on advanced imaging. A recent MRI study in normal volunteers employed fiber tracking to elucidate connectivity among CG25, other DBS depression targets, and their cortical (Brodmann area 10) or limbic (amygdala) network nodes [11]. Preliminary findings in a CG25 DBS depression trial that commenced after closure of the industry trials appear to dovetail with the MRI tractography research. Among 3 sequential study cohorts, targeting in the 2 most recent patient groups employed MRI tractography that was augmented in the third group by intraoperative "repeated bilateral stimulation at the identified optimal contacts for an additional 15-20 min." The combination of tractography-based targeting and prolonged intraoperative stimulation reportedly led to earlyonset antidepressant effects [9].

Another approach entails advanced neuroimaging methods to study DBS depression therapy responders versus nonresponders. In one such study, investigators employed machine learning techniques to analyze baseline and serial post-DBS imaging in TRD subjects implanted in the subcallosal cingulate region (CG25, SCC) [12]. Analyses of serial anatomical (local volumetric MRI) and physiological data (18FDG-PET regional metabolism) separately, and combined, were significantly predictive of DBS responder status. Structural and metabolic changes in serial imaging over time also diverged between DBS responders and nonresponders. The investigators also compared MRI local/regional volumetric data between DBS trial subjects and an imaging archive of healthy normal controls. Post hoc comparisons "... indicated that SCC-DBS responders were generally more similar to controls than non-responders were. Specifically, healthy control relative volumes in the right frontopolar cortex, right superior frontal gyrus, and bilateral orbitofrontal region significantly differed from SCC-DBS non-responder volumes but not from responder volumes. ..." Studies based on TRD-DBS responders raise the matter of control group subjects whose depression scores improved during blinded sham treatment in both trials - as discussed below. Elias et al. [12] noted that it remains to be determined whether advanced imaging techniques will prove as highly predictive in advance as they are during back-testing. The field appears headed toward next-generation studies that employ combinations of volumetric MRI, tractography, and metabolic or other physiological imaging as adjuncts to subject selection and lead targeting. Future selection measures also may explore detailed, multidimensional analyses of patient phenotypes that include domains of cognition, prior experiences, and expectations [12]. The effects of patient expectations also may be germane to findings in the above-mentioned trial where subjects were administered somewhat prolonged intraoperative "stimulation at the identified optimal contacts" [9]. Although speculative, the combination of prolonged stimulation and patient knowledge that it was delivered to the optimal lead contacts could amount to a form of conditioning that, in part, sets future expectations.

Speculation about conditioning and setting expectations also ties in to reports of abrupt relapse or symptom rebound associated with DBS battery depletion. In experiments on the analgesic versus placebo effects of thalamic DBS for pain, investigators studied implanted patients who reported longterm relief of chronic intractable pain [56]. In one experiment, investigators deceived patients to believe that their stimulators were "on" by showing them fake oscilloscope waveforms (a form of misdirection) when, in fact, the devices were "off." In laboratory thermode exposure testing and at-home phases of the study, a majority of the reported analgesic effects were observed in the stimulation "off" compared to "on" setting (74-90\% of the "on" effect, depending on the laboratory or home setting, and whether pain intensity or unpleasantness was rated). Being careful not to apply an inapt analogy, it could be informative and ethically acceptable to study DBS depression (or OCD) patients, especially responders, using similar methods under supervised in-patient conditions.

\section{Summary and Conclusions}

With manufacturers now accruing revenue from DBS devices for psychiatric indications outside the USA - where FDA-approved or unapproved designations ("on-label" or "off-label") do not apply - it remains conjectural whether research advances will lead them to reconsider the investments required to upgrade the current IDE-only status of DBS for depression in the USA, or the HDE for OCD. We have discussed how the low number of potential DBS candidates with severe, treatment-resistant OCD predict- 
ed an insufficient return on investment for sponsors to pursue PMA in the USA. This eventuated in HDE approval, a medical device analog of orphan drug status.

With regard to the TRD trials, the enrollment of subjects with greater comorbidity burdens than anticipated, and the availability of approved (esketamine) and investigational treatments for TRD and suicidality (e.g., lithium, thyroid hormones, atypical antipsychotics, and psychedelic drugs), may prompt a re-evaluation of estimates for greater than 750,000 mood disorder DBS candidates in the USA and tens of millions worldwide [43]. US centers appear to have experienced difficulty recruiting suitable candidates for the DBS trials who did not have confounding psychiatric and/or medical comorbidities. Neuroimaging analyses of CG25 (SCC) DBS responder cohorts with an eye toward refinement of selection criteria more likely will diminish, rather than expand the future TRD candidate pool [12]. Recruitment also has been challenging in an independent CG 25 depression study that commenced in 2014 - after results of both industry trials became public and that applies strict subject selection criteria [57]. The authors attributed some difficulties to hesitancy among potential subjects and referring psychiatrists arising from public knowledge of earlier results. But in light of recent imaging research and responder analyses, a sobering question is whether a sufficient number of optimal candidates could be recruited and retained within sufficient cost and time constraints to make a new, industry-sponsored, PMA scale DBS-TRD trial commercially feasible in the USA. Given the regulatory, historical, and commercial factors discussed here, and in other reviews, guidelines, and consensus statements, physicians and patients in the USA face a near-term future of limited IDE research and HDE status for DBS to treat depression and OCD, respectively. The situation is unlikely to change regardless of whether one frames recommendations in ethical or evidence-based terms - or even if US hospitals, academic institutions, public or private insurance, or medical device manufacturers cover all DBS-related expenses for psychiatric indications [10]. The nature of most evidence to date - despite a growing quantity - is unlikely to qualify for submission to the FDA toward full approval of DBS for clinical use in TRD or OCD.

\section{Acknowledgments}

The authors gratefully acknowledge the collaboration of Andrew Scull, PhD, Distinguished Professor of Sociology and Science Studies at the University of California San Diego, La Jolla, CA for his participation in a symposium entitled: "Psychosurgery in the 21st Century: Evidence-Based or Biased"? presented at the 2019 Annual Meeting of the American Psychiatric Association.

\section{Conflict of Interest Statement}

The authors have no financial conflicts of interest to declare. Dr. R.J. Coffey was an employee of Medtronic, Inc. until January 1,2014 - and served on the Clinical Events Committee for the Reclaim Deep Brain Stimulation Clinical Study for Treatment-Resistant Depression. His employment included participation in the design, conduct, and analysis of all phases of the Reclaim trial program, the physician-sponsored IDE trials leading up to it, and the physician-sponsored IDE studies that led to the OCD HDE. Dr. R.J. Coffey served as the nonvoting Advanced Medical Technology Association (AdvaMed) industry representative on the FDA-CDRH Neurological Devices Advisory panel from 2005 to 2008. Dr. S.N. Caroff has received research grants from Neurocrine Biosciences (San Diego, CA, USA) and Eagle Pharmaceuticals (Woodcliff Lake, NJ, USA), and served as a consultant to Neurocrine Biosciences, Teva Pharmaceuticals (Petah Tikva, Israel), and Adamas Pharmaceuticals (Emeryville, CA, USA).

\section{Funding Sources}

No funding from any source was provided for production of the manuscript.

\section{Author Contributions}

Both authors, Robert J. Coffey, MD and Stanley N. Caroff, MD, contributed equally to the conception, planning, writing, and editing of the manuscript.

\section{References}

1 Mendelsohn D, Lipsman N, Bernstein M. Neurosurgeons' perspectives on psychosurgery and neuroenhancement: a qualitative study at one center. J Neurosurg. 2010;113:1212-8.

2 Scull A. Desperate remedies. Embrace of a deadly cure. Princeton Alumni Weekly. 2005 May 11: Feature. Available from: http://www. princeton.edu/ paw/archive_new/PAW0405/14-0511/features1.html.
3 Caroff S. Depression, other behavioral disorders, and DBS: furor therapeuticus redux. Clin Psych News. 2017 Apr:12. Available from: https: //www.mdedge.com/psychiatry/article/ 132967/depression/depression-and-deep-brainstimulation-furor-therapeuticus-redux.

4 Dougherty DD, Rezai AR, Carpenter LL, Howland RH, Bhati MT, O'Reardon JP, et al. A randomized sham controlled trial of deep brain stimulation of the ventral capsule/ventral striatum for chronic treatment-resistant depression. Biol Psychiatry. 2015;78:240-8.

5 Holtzheimer PE, Husain MM, Lisanby SH, Taylor SF, Whitworth LA, McClintock S, et al. Subcallosal cingulate deep brain stimulation for treatment-resistant depression: a multisite, randomised, sham-controlled trial. Lancet Psychiatry. 2017;4:839-49.
Continued Investigational Status of DBS

for Psychiatric Indications
Stereotact Funct Neurosurg 2022;100:156-167 DOI: $10.1159 / 000521395$ 
6 A future for neuromodulation in psychiatric disease. Stereotact Funct Neurosurg. 2015;93: 69.

7 Widge AS, Malone DA, Dougherty DD. Closing the loop on deep brain stimulation for treatment-resistant depression. Front Neurosci. 2018; 12:175.

8 Peña C, Bowsher K, Costello A, De Luca R, Doll S, Li K, et al. An overview of FDA medical device regulation as it relates to deep brain stimulation devices. IEEE Trans Neural Syst Rehabil Eng. 2007;15:421-4.

9 Riva-Posse P, Crowell AL, Wright K, Waters AC, Choi KS, Garlow SJ, et al. Rapid antidepressant effects of deep brain stimulation and their relation to surgical protocol. Biol Psych. 2020;88:e37-9.

10 Vedam-Mai V, Deisseroth K, Giordano J, Lazaro-Munoz G, Chiong W, Suthana N, et al. Proceedings of the eighth annual deep brain stimulation think tank: advances in optogenetics, ethical issues affecting DBS research, neuromodulatory approaches for depression, adaptive neurostimulation, and emerging DBS technologies. Front Hum Neurosci. 2021;15: 644593.

11 Zhu Z, Hubbard E, Guo X, Barbosa DAN, Popal AM, Cai C, et al. A connectomic analysis of deep brain stimulation for treatment resistant depression. Brain Stimul. 2021;14:1226-33.

12 Elias GJB, Germann J, Boutet A, Pancholi A, Beyn ME, Bhatia K, et al. Structuro-functional surrogates of response to subcallosal cingulate deep brain stimulation for depression. Brain. 2021. Epub ahead of print. Available from: https: //academic.oup.com/brain/advancearticle-abstract/doi/10.1093/brain/awab284/ 6330761? redirectedFrom=fulltext.

13 Nuttin B, Cosyns P, Demeulemeester H, Gybels J, Meyerson B. Electrical stimulation in anterior limbs of internal capsules in patients with obsessive-compulsive disorder. Lancet. 1999;354:1526.

14 Malone DA, Dougherty DD, Rezai AR, Carpenter LL, Friehs GM, Eskandar EE, et al. Deep brain stimulation of the ventral capsule/ ventral striatum for treatment-resistant depression. Biol Psychiatry. 2009;65:267-75.

15 Rauch SL, Dougherty DD, Malone D, Rezai A, Friehs G, Fischman AJ, et al. A functional neuroimaging investigation of deep brain stimulation in patients with obsessive-compulsive disorder. J Neurosurg. 2006;104:558-65.

16 Bergfeld IO, Mantione M, Hoogendoorn MLC, Ruhé HG, Notten P, van Laarhoven J, et al. Deep brain stimulation of the ventral anterior limb of the internal capsule for treatmentresistant depression. a randomized clinical trial. JAMA Psychiatry. 2016;73:456-64.

17 Bentzley BS, Pannu J, Badran BW, Halpern $\mathrm{CH}$, Williams NR. It takes time to tune. Ann Transl Med. 2017;5:171.

18 Youngerman BE, Sheth SA. Deep brain stimulation for treatment-resistant depression: optimizing interventions while preserving valid trial design. Ann Transl Med. 2017; 5(Suppl 1):S1.
19 Bewernick BH, Hurlemann R, Matusch A, Kayser S, Grubert C, Hadrysiewicz B, et al. Nucleus accumbens deep brain stimulation decreases ratings of depression and anxiety in treatment-resistant depression. Biol Psych. 2010;67:110-6.

20 Bewernick BH, Kayser S, Volker Sturm V, Schlaepfer TE. Long-term effects of nucleus accumbens deep brain stimulation in treatmentresistant depression: evidence for sustained efficacy. Neuropsychopharmacology. 2012;37: 1975-85.

21 Montgomery SA, Asberg M. A new depression scale designed to be sensitive to change. $\mathrm{Br} \mathrm{J}$ Psychiatry. 1979;134:382-9.

22 Hamilton M. A rating scale for depression. J Neurol Neurosurg Psychiatry. 1960;23:56-62.

23 Lozano AM, Giacobbe P, Hamani C, Rizvi SJ, Kennedy SH, Kolivakis TT, et al. A multicenter pilot study of subcallosal cingulate area deep brain stimulation for treatment-resistant depression. J Neurosurg. 2012;116:315-22.

24 Dunner DL, Rush AJ, Russell JM, Burke M, Woodard S, Wingard P, et al. Prospective, longterm, multicenter study of the naturalistic outcomes of patients with treatment-resistant depression. J Clin Psychiatry. 2006;67:688-95.

25 Mayberg HS, Lozano AM, Voon V, McNeely HE, Seminowicz D, Hamani C, et al. Deep brain stimulation for treatment-resistant depression. Neuron. 2005;45:651-60.

26 Hamani C, Mayberg H, Snyder B, Giacobbe P, Kennedy S, Lozano AM. Deep brain stimulation of the subcallosal cingulate gyrus for depression: anatomical location of active contacts in clinical responders and a suggested guideline for targeting. J Neurosurg. 2009;111:1209-15.

27 Fisher R, Salanova V, Witt T, Worth R, Henry $\mathrm{T}$, Gross R, et al. Electrical stimulation of the anterior nucleus of thalamus for treatment of refractory partial epilepsy. Epilepsia. 2010;51: 899-908.

28 Salanova V. Deep brain stimulation for epilepsy. Epilepsy Behav. 2018;88S:21-4.

29 Fins JJ, Mayberg HS, Nuttin B, Kubu CS, Galert T, Sturm V, et al. Misuse of the FDAs humanitarian device exemption in deep brain stimulation for obsessive-compulsive disorder. Health Aff. 2011;30:302-11.

30 Pinckard-Dover H, Ward H, Foote KD. The decline of deep brain stimulation for obsessivecompulsive disorder following FDA humanitarian device exemption approval. Front Surg. 2021;8:642503.

$31 \mathrm{Wu} \mathrm{H}$, Hariz M, Visser-Vandewalle V, Zrinzo L, Coenen VA, Sheth SA, et al. Deep brain stimulation forrefractory obsessive-compulsive disorder (OCD): emerging or established therapy? Mol Psychiatry. 2021;26:60-5.

32 Denys D, Graat I, Mocking R, de Koning P, Vulink N, Figee M, et al. Efficacy of deep brain stimulation of the ventral anterior limb of the internal capsule for refractory obsessivecompulsive disorder: a clinical cohort of 70 patients. Am J Psychiatry. 2020;177:265-71.

33 Goodman WK, Storch EA, Cohn JF, Sheth SA. Deep brain stimulation for intractable obsessive- compulsive disorder: progress and opportunities. Am J Psychiatry. 2020;177:200-3.

34 Hamani C, Pilitsis J, Rughani AI, Rosenow JM, Patil PG, Slavin KS, et al. Deep brain stimulation for obsessive-compulsive disorder: systematic review and evidence-based guideline sponsored by the American Society for stereotactic and functional neurosurgery and the Congress of Neurological Surgeons (CNS) and endorsed by the CNS and American Association of Neurological Surgeons. Neurosurgery. 2014;75:327-33.

35 Luyten L, Hendrickx S, Raymaekers S, Gabriëls L, Nuttin B. Electrical stimulation in the bed nucleus of the stria terminalis alleviates severe obsessive-compulsive disorder. Mol Psychiatry. 2016;21:1272-80.

36 Denys D, Mantione M, Figee M, van den Munckhof P, Koerselman F, Westenberg H, et al. Deep brain stimulation of the nucleus accumbens for treatment-refractory obsessivecompulsive disorder. Arch Gen Psychiatry. 2010;67:1061-8.

37 Mallet L, Polosan M, Jaafari N, Baup N, Welter ML, Fontaine D, et al. Subthalamic nucleus stimulation in severe obsessive-compulsive disorder. N Engl J Med. 2008;13359:2121-34.

38 Menchón JM, Real E, Alonso P, Aparicio MA, Segalas C, Plans G, et al. A prospective international multi-center study on safety and efficacy of deep brain stimulation for resistant obsessive-compulsive disorder. Mol Psych. 2021;26:1234-47.

39 Mosley PE, Windels F, Morris J, Coyne T, Marsh R, Giorni A, et al. A randomised, double-blind, sham-controlled trial of deep brain stimulation of the bed nucleus of the stria terminalis for treatment-resistant obsessive-compulsive disorder. Transl Psychiatry. 2021;11:190207.

40 Tyagi H, Apergis-Schoute AM, Akram H, Foltynie T, Limousin P, Drummond LM, et al. A randomized trial directly comparing ventral capsule and anteromedial subthalamic nucleus stimulation in obsessive-compulsive disorder: clinical and imaging evidence for dissociable effects. Biol Psychiatry. 2019;85:726-34.

41 Nuttin B, Wu H, Mayberg H, Hariz M, Gabriëls $\mathrm{L}, \mathrm{Galert} \mathrm{T}$, et al. Consensus on guidelines for stereotactic neurosurgery for psychiatric disorders. J Neurol Neurosurg Psychiatry. 2014;85: 1003-8.

42 Fineberg NA, Hollanderd E, Pallantie S, Walitzag S, Grünblattg E, Dell'Osso BM, et al. Clinical advances in obsessive-compulsive disorder: a position statement by the International College of Obsessive Compulsive Spectrum Disorders. Int Clin Psychopharmacol. 2020;35: 173-93.

43 World Health Organization. The global burden of disease: 2004 update. Geneva, Switzerland: WHO Press; 2008. p. 160. Available from: https://scholar.google.com/scholar_lookup? title $=$ The $\% 20$ Global\%20Burden $\% 20$ of $\% 20$ Disease $\% 3$ A \%202004\%20Update\& author=World\%20Health\%20Organization\& publication_year $=2008$. 
44 Gaynes BN, Rush AJ, Trivedi MH, Wisniewski SR, Spencer D, Fava M. The STAR*D study: treating depression in the real world. Cleve Clin J Med. 2008;75:57-66.

45 Thomson W. Long term follow up of suicide in a clinically depressed community sample. J Affect Disord. 2012;139:52-5.

46 Gronemann FH, Jørgensen MB, Nordentoft M, Andersen PK, Osler M. Treatment-resistant depression and risk of all-cause mortality and suicidality in Danish patients with major depression. J Psychiatr Res. 2021;135:197202.

47 Bergfeld IO, Mantione M, Figee M, Schuurman PR, Lok A, Denys D. Treatment-resistant depression and suicidality. J Affect Disord. 2018;235:362-7.

48 Varkey B. Principles of clinical ethics and their application to practice. Med Princ Pract. 2021;30(1):17-28.
49 Cardozo B. Basic right to consent to medical care - Schlendorff vs. the Society of the New York Hospital, 211 NY 125, 105 NE 92, 1914 LEXUS 1028. 1914

50 Cookson R, Dolan P. Principles of justice in health care rationing. J Med Ethics. 2000;26: 323-9.

51 American Psychiatric Association. Diagnostic and statistical manual of mental disorders. 4th edn. Text revision. Washington, DC: American Psychiatric Association; 2000.

52 American Psychiatric Association. Diagnostic and statistical manual of mental disorders. 5th edn. Arlington, VA: American Psychiatric Association; 2013.

53 Bernstein L. Addiction treatment had failed. Could brain surgery save him? The Washington Post. 2021 Jun 18. Available from: https://www. washingtonpost.com/health/2021/06/18/deepbrain-stimulation-addiction/.
54 Bentzley BS, Pannu J, Badran BW, Halpern $\mathrm{CH}$, Williams NR. It takes time to tune. Ann Transl Med. 2017;5:171.

55 McIntyre RS, Rosenblat JD, Nemeroff CB, Sanacora G, Murrough JW, Berk M, et al. Synthesizing the evidence for ketamine and esketamine in treatment-resistant depression: an international expert opinion on the available evidence and implementation. Am J Psychiatry. 2021;178(5):383-99.

56 Marchand S, Kupers RC, Bushnell CM, Duncan GH. Analgesic and placebo effects of thalamic stimulation. Pain. 2003;105(3): 481-8.

57 Ramasubbu R, Golding S, Williams K, Mackie A, MacQueen G, Kiss ZHT. Recruitment challenges for studies of deep brain stimulation for treatment-resistant depression. Neuropsychiatr Dis Treat. 2021;17:76575 . 